Revista Brasileira de Higiene e Sanidade Animal Brazilian Journal of Hygiene and Animal Sanity

ISSN: 1981-2965

\title{
Avaliação morfológica de espermatozoides suínos em uma central de inseminação artificial
}

Morfological sperm evaluation in boars from an artificial insemination center

\begin{abstract}
Victória Pontes Rocha ${ }^{1}$, Lina Raquel Santos Araújo ${ }^{2^{*}}$, Luiz Antônio Moreira Miranda ${ }^{3}$, Beatriz Mano e Silva ${ }^{4}, \hat{E}$ nio Campos da Silva ${ }^{5}$, Fágner Cavalcante Patrocínio dos Santos ${ }^{6}$
\end{abstract}

Resumo: Alterações na morfologia espermática têm impacto sobre a fertilidade de reprodutores suínos, com prejuízos nos índices produtivos, constituindo uma das causas para descarte de machos. Nesse contexto, objetivou-se identificar anormalidades mais frequentes em amostras de sêmen de cachaços adultos que foram reprovadas, em uma central de inseminação, além de prospectar considerações acerca do descarte de reprodutores baseado no exame morfológico. Para atingir tal objetivo foram coletados o sêmen de 95 suínos e avaliada a morfologia espermática. Dentre os 95 reprodutores que tiveram o sêmen avaliado na primeira coleta, $78 \%$ tiveram o sêmen aprovado e $22 \%$ reprovado. Durante a primeira coleta de sêmen, do total de anormalidades espermáticas $(31,52 \% \pm 8,85)$ as mais frequentes foram: gota citoplasmática distal $(13,76 \% \pm 7,48)$ e proximal $(11,95 \% \pm 6,72)$. Os 21 reprodutores com amostras reprovadas na primeira coleta, foram novamente avaliados e 19\% (4 reprodutores) reprovados, novamente do total de anormalidades observadas $(33,75 \% \pm 2,38)$, destacou-se a gota citoplasmática distal $(14,25 \% \pm 1,92)$ e proximal $(12,5 \% \pm 4,39)$. Através do presente estudo foi possível observar que a avaliação morfológica de espermatozoides em granjas suínas é uma ferramenta extremamente necessária, para identificar animais para seleção ou descarte reprodutivo. A repetição do exame morfológico em machos que tiveram o sêmen reprovado, deve ser realizada a fim de evitar falsas conclusões.

Palavras-chave: Fertilidade. Patologia espermática. Suinocultura.

Abstract: Changes in sperm morphology have an impact on the fertility of swine reproducers, with losses in the productive indexes, constituting one of the causes of discarding males. In this context, the objective was to identify more frequent abnormalities in semen samples from boars that failed, at an insemination center, in addition to exploring considerations about the disposal of breeders based on the morphological examination. To achieve this goal, semen was collected from 95 pigs and sperm morphology was evaluated. Among the 95 breeders that had their semen evaluated in the first collection, $78 \%$ had their semen approved and $22 \%$ failed. During the first semen collection, most of the sperm abnormalities $(31.52 \% \pm 8.85)$ identified were distal $(13.76 \% \pm 7.48)$ and proximal $(11.95 \%$ \pm 6.72 ) cytoplasmic gout. The 21 breeders with failed samples in the first collection, were again evaluated and $19 \%$ failed (4 boars), the average recurrent abnormalities $(33.75 \% \pm 2.38)$ were distal $(14.25 \% \pm 1.92)$ and proximal $(12.5 \% \pm 4.39)$ cytoplasmic gout. Through this study it was possible to observe that the morphological evaluation of sperm in swine farms is an extremely necessary tool to identify animals for selection and reproductive disposal. The repetition of the morphological examination in males that had their semen failed should be performed in order to avoid false conclusions.

Index terms: Fertility. Pig farming. Sperm pathology. 


\section{http://dx.doi.org/}

Autora para correspondência, e-mail: * lina.araujo@professor.unifametro.edu.br

Recebido em 11.03.2021. Aceito em 30.06.2021

1 Médica Veterinária, Mestranda em Ciência Animal, Escola de Veterinária, Universidade Federal de Minas

Gerais, Av. Antônio Carlos, 6627, Caixa Postal 567, 31.270-901, Belo Horizonte, MG. E-mail: mvvictoriapr@gmail.com

2 Médica Veterinária, Professora, Centro Universitário Fametro, Curso de Medicina Veterinária, Rua Carneiro da

Cunha, 180, Jacarecanga, 60.010-470, Fortaleza, CE. E-mail: lina.araujo@professor.unifametro.edu.br

3 Graduando em Medicina Veterinária, Faculdade de Veterinária, Universidade Estadual do Ceará, Av. Silas

Munguba, 1700, Itaperi, 60.714-903, Fortaleza, CE. E-mail: luiz.antonio@ @aluno.uece.br

4 Graduanda em Medicina Veterinária, Faculdade de Veterinária, Universidade Estadual do Ceará, Av. Silas

Munguba, 1700, Itaperi, 60.714-903, Fortaleza, CE. E-mail: beatriz.mano@aluno.uece.br

5 Médico Veterinário, Professor, IFOPE Soluções e Treinamento, Rua Gonçalves Dias, 55, Funcionários, 30.140-

090, Belo Horizonte, MG. E-mail: eniocampospdisco@ hotmail.com

6 Professor Substituto, Faculdade de Veterinária, Universidade Estadual do Ceará, Av. Silas Munguba, 1700, Itaperi, 60.714-903, Fortaleza, CE. E-mail: fagner.cavalcante@uece.br

\section{Introdução}

A inseminação artificial (IA) é uma biotécnica amplamente utilizada na espécie suína, nas últimas décadas, as granjas de grande e médio porte utilizam tal atividade agregando diversos benefícios aos sistemas de produção suinícolas no Brasil. Por apresentar uma variedade de vantagens para suinocultura, a IA agrega redução dos custos de produção, garante uma melhor segurança e controle sanitário nos plantéis, eleva os índices de eficiência reprodutiva dos animais e representa avanços, tecnificação e modernização em torno da produção de carne suína.

Dentre os pontos cruciais para adotar a IA nos sistemas de produção suinícolas, destaca-se a redução no número de machos necessários à reprodução e, consequentemente, redução dos custos com ração, medicamentos, instalações e mão de obra durante os manejos (TONIOLLI, 2002; BORTOLOZZO et al., 2005; KNECHT et al., 2017). Assim, o cachaço assume uma maior responsabilidade em termo do número de descendentes e a infertilidade ou subfertilidade é um fator limitante na produção e escolha do animal.

Para a seleção de reprodutores suínos em uma Central de Produção de Sêmen (CPS), muitos parâmetros devem ser avaliados antes e após admissão do macho para produção de doses de sêmen, como a produção e a qualidade espermática, 
parâmetros constantemente avaliados para garantir a qualidade e a produção das doses

inseminantes (MELLAGI et al., 2019).

A reposição anual de machos em uma CPS é comumente acima de $80 \%$, o que permite utilização de doses inseminantes provenientes de reprodutores com índices genéticos constantemente atualizados. Assim, periodicamente reprodutores são descartados, dentre as principais causas de descarte estão o melhoramento genético e a baixa qualidade espermática, tornando a análise seminal de suínos primordial para a otimização desse processo (ARRUDA et al., 2015; ALKMIN, 2019; BUSTAMANTE-FILHO et al., 2019).

A análise espermática é o procedimento de identificação dos componentes do espermatozoide, a fim de quantificar e qualificar anormalidades para classificar os reprodutores em aptos ou não aptos à reprodução. É comum observar no sêmen de cachaços saudáveis uma certa população de espermatozoides morfologicamente anormais, porém, distúrbios na fertilidade do animal podem acontecer quando essa população ultrapassa um limite pré-estabelecido que corresponde a valores superiores a $20 \%$ (JUNG et al., 2015; MELLAGI et al., 2019).
Os defeitos espermáticos podem ou não estar relacionados a fatores genéticos, as anormalidades de origem genética são inespecíficas e raras, ocasionando redução acentuada da fertilidade ou até mesmo à esterilidade (BRIZ et al., 1995; BORTOLOZZO et al., 2005; GAGGINI et al., 2018). Outros defeitos aparecem após a saída dos espermatozoides dos testículos, tem origem no epidídimo, durante $\mathrm{o}$ processo de maturação e permanecem até o momento da ejaculação, sendo representadas, principalmente, pela ocorrência de gota citoplasmática devido à maturação incompleta (BRACKETT, 2006; BONET et al., 2012; OBERLENDER et al., 2013; ARRUDA et al, 2015; GAGGINI et al., 2018).

Cabeças de espermatozoides e acrossoma destacados, caudas dobradas e anormalidades da peça intermediária/cauda também são causadas por fatores externos como traumatismo, exposição a temperaturas elevadas e patógenos (Bortolozzo et al., 2005; Arruda et al., 2015). Algumas irregularidades espermáticas são causadas pela manipulação humana inadequada e mudanças ambientais durante $\mathrm{O}$ processamento do sêmen ou na escolha da técnica de avaliação morfológica. Como não há influência na fertilidade do sêmen 
avaliado, para que não haja conclusões errôneas, é necessário que o procedimento seja repetido com um novo ejaculado (BORTOLOZZO et al., 2005; BONET et al., 2012).

Adicionalmente, percentuais considerados altos de anormalidades podem ser permanentes ou temporários, dependendo da causa. Os resultados de morfologia espermática podem ajudar na identificação de alterações reprodutivas que possam resultar em problemas de fertilidade (BRACKETT, 2006; MELLAGI et al., 2019), além de auxiliar na escolha e descarte de animais para as centrais de IA.

Dessa forma, o objetivo do presente estudo foi avaliar as principais anormalidades identificadas em amostras de sêmen suíno reprovadas em uma central de inseminação, além de realizar considerações acerca do descarte de reprodutores baseado apenas em um exame morfológico.

\section{Materiais e Métodos}

O estudo foi realizado em uma Central de Inseminação Artificial de Suínos localizada no estado do Rio Grande do Sul. Foi objeto de estudo os resultados dos exames morfológicos das amostras dos ejaculados dos 95 machos ativos da central, com idade variando entre 8 e 26 meses (média 14,2 meses), da linhagem genética Agroceres PIC®. As coletas de sêmen foram realizadas em julho de 2019, durante o período da manhã, através do método da mão enluvada (GONZALEZCADAVID et al., 2014).

Primeiramente foi realizada a higienização do prepúcio dos animais e a coleta realizada com o auxílio de um copo previamente aquecido $\left(35^{\circ} \mathrm{C}\right)$, revestido internamente por saco plástico coletor adaptado com papel filtro, para separação das frações gelatinosa e líquida do ejaculado. Tais animais eram submetidos à coleta de sêmen para fabricação de doses inseminantes, semanalmente.

Após a coleta de sêmen, $300 \mu \mathrm{L}$ (três gotas) de sêmen in natura foram armazenados em microtubos pré-aquecido, contendo $2 \mathrm{~mL}$ de formol a 2,94\% (formol citrato). Posteriormente, as amostras foram refrigeradas a $17{ }^{\circ} \mathrm{C}$, armazenadas em recipiente térmico para transporte e enviadas ao laboratório análises morfológicas. Nas análises foram confeccionados esfregaços seminais corados contendo uma gota de sêmen e uma gota de corante eosinanigrosina, após esta etapa, foi feita a contagem das células com auxílio de microscópio óptico e contador de células. Foram contadas 200 células em cada amostra e dentre elas, foram classificadas aquelas com alteração de cabeça, acrossoma, colo, peça intermediária e cauda. 
O sêmen dos machos foi classificado em aprovado ou reprovado para a fabricação de doses inseminantes, tendo como critério as anormalidades espermáticas. Para ser considerada aprovada, a amostra avaliada deveria ter percentual inferior a $30 \%$ de espermatozoides com alterações morfológicas, sendo que se analisados individualmente, o total aceitável era de até 5\% para patologias primárias (defeitos na cabeça, colo, acrossoma e peça intermediária) e $10 \%$ para patologias secundárias (gota citoplasmática e defeitos de cauda dobrada ou enrolada). Se houvesse a reprovação em alguma das amostras, o recomendado era esgotar o macho semanalmente, respeitando um intervalo de 7 dias, no mínimo. Porém, esse ejaculado não seria utilizado durante 3 semanas, sendo enviado para reavaliação, no mês seguinte.

Se houvesse novamente a reprovação da amostra de sêmen do mesmo animal, este animal seria avaliado quanto a idade, o índice genético, o comportamento sexual e aprumos, para então ser descartado. A meta de reposição anual da CPSS é de $100 \%$.

Os dados obtidos foram analisados através do método de estatística descritiva, que mostra todos os dados brutos obtidos no estudo, de forma simples, realista e direta (TURNER \& HOULE, 2019). Os parâmetros morfológicos analisados foram apresentados em valores percentuais, para cada anormalidade identificada, para as amostras reprovadas na primeira e na segunda coleta. Os dados de média e desvio padrão para as anormalidades identificadas foram agrupados e gerados através de planilhas do Excel.

\section{Resultados e Discussão}

No presente estudo foram utilizadas técnicas de coleta de sêmen e avaliação morfológica espermática por microscopia, para identificar defeitos seminais em suínos que os inviabilizam de permanecer como doadores seminais, em centrais de IA. Utilizou-se métodos de estatística descritiva, para observar as principais anormalidades e orientar o descarte animal, após 2 coletas e 2 avaliações. Identificar animais com anormalidades severas é um fator imprescindível para a seleção de reprodutores, uma vez que manter em centrais animais de baixa qualidade espermática, acarreta prejuízos econômicos aos suinocultores e pode gerar produtos, nas próximas gerações, de má qualidade, além do repasse de tais característica nas gerações seguintes, quando é um fator de origem genética. 
Dentre os 95 reprodutores que tiveram o sêmen avaliado, $78 \%$ tiveram o sêmen aprovado com base nos percentuais de patologias encontradas no exame morfológico, enquanto $22 \%$ dos reprodutores tiveram o sêmen reprovado.
Os animais com sêmen reprovado (aqueles que possuíam mais de $30 \%$ de anormalidades totais, ou um percentual maior que $10 \%$ de patologias individuais) têm seus percentuais das anormalidades dispostos por tipo apresentados na Tabela 1.

Tabela 1. Percentuais das anormalidades encontradas nas amostras de sêmen reprovadas ( $1^{\mathrm{a}}$ coleta).

\begin{tabular}{|c|c|c|c|c|c|c|c|}
\hline Macho & $\begin{array}{c}\text { Total de } \\
\text { anomalia } \\
\text { s (\%) }\end{array}$ & $\begin{array}{c}\text { Cabeça } \\
\text { isolada } \\
(\%)\end{array}$ & $\begin{array}{c}\text { Cauda } \\
\text { dobrada/ } \\
\text { Enrolada } \\
\quad(\%)\end{array}$ & $\begin{array}{c}\text { Cauda } \\
\text { dobrada } \\
\text { com } \\
\text { GCP* } \\
(\%)\end{array}$ & $\begin{array}{c}\text { Cauda } \\
\text { dobrada } \\
\text { com } \\
\text { GCD** } \\
(\%)\end{array}$ & $\begin{array}{c}\text { GCP* } \\
(\%)\end{array}$ & $\begin{array}{c}\text { GCD }^{* *} \\
(\%)\end{array}$ \\
\hline 01 & 20 & 0 & 0 & 0 & 2 & 16 & 2 \\
\hline 02 & 25 & 0 & 0 & 0 & 1 & 19 & 5 \\
\hline 03 & 28 & 0 & 0 & 0 & 3 & 21 & 4 \\
\hline 04 & 30 & 0 & 1 & 0 & 2 & 3 & 24 \\
\hline 05 & 30 & 0 & 0 & 0 & 1 & 20 & 9 \\
\hline 06 & 30 & 0 & 0 & 0 & 2 & 5 & 23 \\
\hline 07 & 32 & 0 & 0 & 0 & 7 & 6 & 19 \\
\hline 08 & 32 & 0 & 0 & 0 & 0 & 27 & 5 \\
\hline 09 & 33 & 0 & 0 & 1 & 1 & 17 & 14 \\
\hline 10 & 33 & 0 & 0 & 1 & 5 & 9 & 18 \\
\hline 11 & 38 & 0 & 0 & 0 & 7 & 10 & 21 \\
\hline 12 & 45 & 0 & 0 & 2 & 10 & 10 & 23 \\
\hline 13 & 46 & 0 & 1 & 1 & 7 & 20 & 17 \\
\hline 14 & 57 & 0 & 9 & 0 & 6 & 13 & 29 \\
\hline 15 & 31 & 0 & 3 & 1 & 15 & 4 & 8 \\
\hline 16 & 27 & 0 & 0 & 1 & 2 & 9 & 15 \\
\hline 17 & 26 & 0 & 0 & 0 & 2 & 10 & 14 \\
\hline 18 & 25 & 0 & 0 & 0 & 3 & 9 & 13 \\
\hline 19 & 24 & 0 & 0 & 1 & 6 & 7 & 10 \\
\hline 20 & 17 & 0 & 0 & 0 & 4 & 1 & 12 \\
\hline 21 & 33 & 3 & 0 & 2 & 9 & 15 & 4 \\
\hline $\begin{array}{c}\text { Média } \pm \\
\text { DP }\end{array}$ & $31,5 \pm 8,8$ & $0,1 \pm 0,6$ & $0,7 \pm 2,0$ & $0,5 \pm 0,76$ & $4,5 \pm 3,6$ & $11,9 \pm 6,7$ & $13,8 \pm 7,5$ \\
\hline
\end{tabular}

*Gota citoplasmática proximal. **Gota citoplasmática distal. DP: desvio padrão.

Através do presente estudo foram identificadas na primeira coleta de sêmen anormalidades espermáticas $(31,52 \% \pm$ $8,85)$, em sua maioria, classificadas como gota citoplasmática distal $(13,76 \% \pm 7,48)$ e proximal $(11,95 \% \pm 6,72)$, conforme descrito também na Tabela 1. Alguns dos principais defeitos podem ser observados na Figura 1.

Os 21 reprodutores com amostras reprovadas na primeira coleta, foram novamente avaliados, após o período de três semana, e foi reprovado um total de 4 animais (19\%). 
Figura 1. Principais anormalidades observadas nas células espermáticas de suínos reprovados para doses inseminantes em uma central de inseminação artificial.

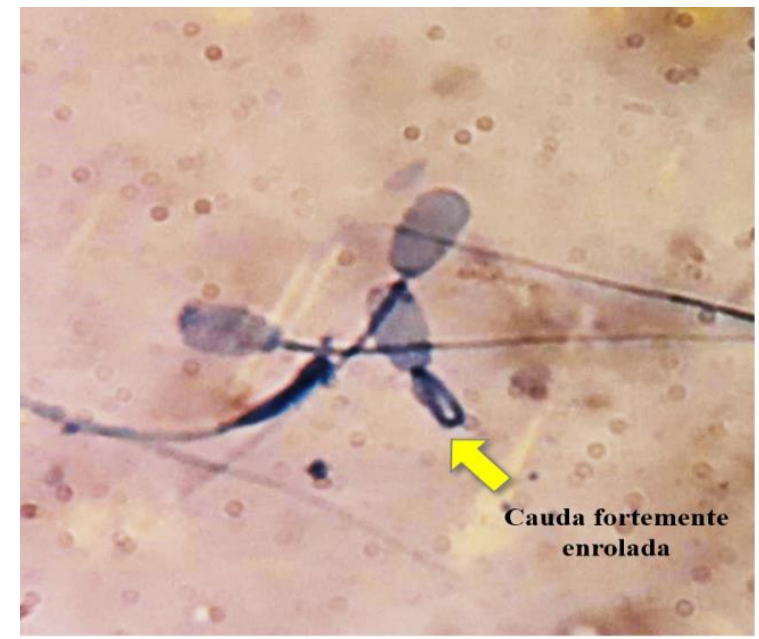

A Figura 2 representa o total de amostra dos animais avaliados na primeira coleta de sêmen (gráfico azul e laranja) e

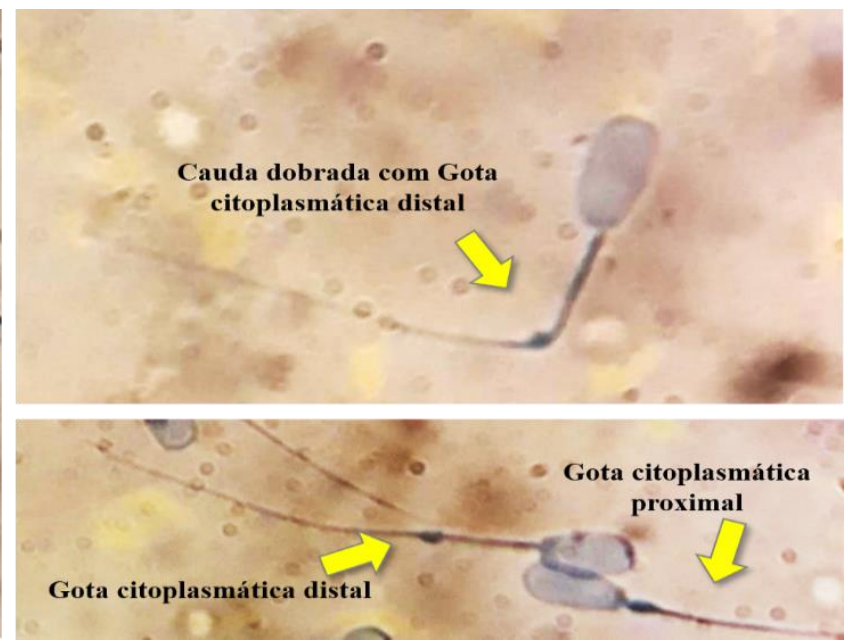

as reprovadas seguiram para uma nova avaliação.

Figura 2. Percentuais de amostras de sêmen suíno aprovadas e reprovadas em uma central de inseminação artificial (gráfico azul e laranja), em um primeiro momento, e animais reprovados

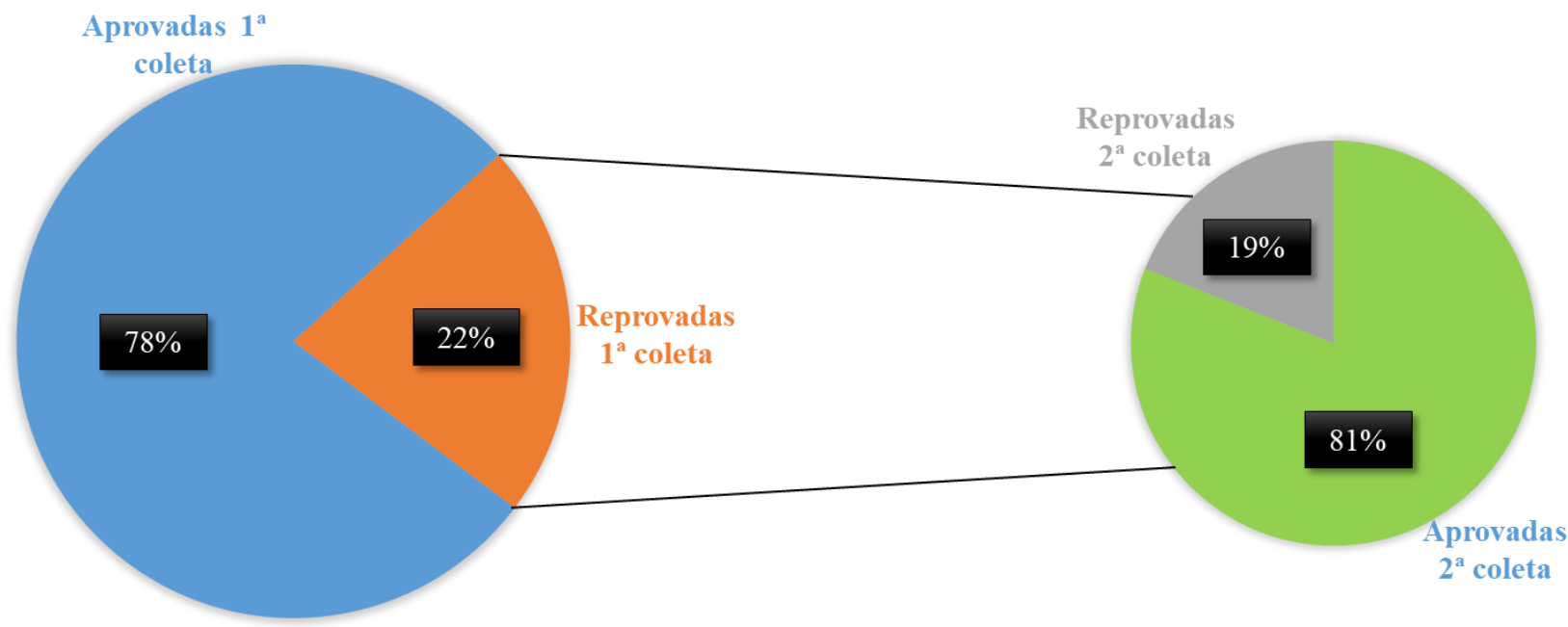

seguiram para uma nova avaliação, após três semanas (gráfico verde e cinza).

$\mathrm{Na}$ segunda coleta (Figura 2 gráfico verde e cinza), a maioria dos animais dos animais tiveram as amostras aprovados pelo teste morfológico (81\%), 
enquanto apenas $19 \%$ foram reprovados e apresentaram como anormalidades médias mais recorrentes a gota citoplasmática
$(14,25 \% \pm 1,92)$ distal e proximal $(12,5 \%$ $\pm 4,39)$.

Tabela 2. Percentuais das anormalidades observadas nas amostras de sêmen reprovadas.

\begin{tabular}{|c|c|c|c|c|c|c|c|}
\hline Macho & $\begin{array}{c}\text { Total de } \\
\text { anomalia } \\
\text { s } \\
(\%)\end{array}$ & $\begin{array}{c}\text { Cabeça } \\
\text { isolada } \\
(\%)\end{array}$ & $\begin{array}{c}\text { Cauda } \\
\text { dobrada/ } \\
\text { enrolada } \\
(\%)\end{array}$ & $\begin{array}{c}\text { Cauda } \\
\text { dobrada } \\
\text { com } \\
\text { GCP* } \\
(\%)\end{array}$ & $\begin{array}{c}\text { Cauda } \\
\text { dobrada } \\
\text { com } \\
\text { GCD** } \\
(\%)\end{array}$ & $\begin{array}{c}\text { GCP* } \\
(\%)\end{array}$ & $\begin{array}{c}\text { GCD }^{* *} \\
(\%)\end{array}$ \\
\hline 07 & 32 & 0 & 3 & 1 & 2 & 9 & 17 \\
\hline 12 & 35 & 0 & 0 & 0 & 0 & 20 & 15 \\
\hline 15 & 37 & 0 & 1 & 2 & 10 & 11 & 13 \\
\hline 18 & 31 & 0 & 0 & 1 & 8 & 10 & 12 \\
\hline $\begin{array}{c}\text { Média } \pm \\
\text { DP }\end{array}$ & $33,7 \pm 2,4$ & $0,0 \pm 0,0$ & $2,0 \pm 1,0$ & $1,3 \pm 0,5$ & $6,7 \pm 3,4$ & $12,5 \pm 4,4$ & $14,2 \pm 1,9$ \\
\hline
\end{tabular}

*Gota citoplasmática proximal. **Gota citoplasmática distal. DP: desvio padrão.

Os percentuais de anormalidades encontradas nas amostras de sêmen dos animais que foram descartados, se encontraram acima de 30\%, como preconizado pelo CBRA (2013). Prejuízos na fertilidade do animal podem ocorrer, quando a população de espermatozoides anormais ultrapassa um limite estabelecido (Jung et al., 2015), já que raramente são encontrados espermatozoides com anomalias na junção útero-tubária (GARCÍA-VÁZQUEZ, et al. 2015) e, assim, sendo atípicos no local de fertilização.

Não foram identificadas irregularidades primárias nas amostras analisadas no estudo. Todavia, foi verificada a ocorrência de anormalidades secundárias, como cabeça isolada e cauda dobrada/enrolada, que podem também ser de origem terciária conforme afirma BORTOLOZZO et al. (2005). Na fase préanalítica de processamento do ejaculado em que se concentram a maior frequência de erros de manipulação, fatores como confecção inadequada do esfregaço, mudanças bruscas de temperatura e $\mathrm{pH}$ e contaminação bacteriana afetam diretamente nos índices de anormalidades espermáticas presentes na amostra coletada (RODRIGUEZ, 2017; SERTÃO, 2019). 
Dentre as irregularidades secundárias encontradas nas amostras, as que mais se destacaram foram as gotas citoplasmáticas proximal e distal (Tabelas 1 e 2; Figuras 1 e 2), corroborando com os resultados obtidos por OBERLENDER et al. (2013) e CAPELETTO et al. (2014), que consideram essas normalidades espermáticas como as mais prevalentes na espécie suína.

A ocorrência de níveis elevados de gota citoplasmática possui correlação negativa com a fertilidade (WABERSKI et al., 1994), embora o mecanismo através do qual a gota ocasiona prejuízo na fertilidade do macho ainda não esteja completamente elucidado (CAPELETTO et al., 2014). Há estudos que apontam que o acontecimento de gotas citoplasmáticas distais em um grande número de espermatozoides, culmina no detrimento da motilidade destes (GAGGINI et al., 2018). Uma elevada frequência de gota citoplasmática distal influencia negativamente a taxa de parto e o tamanho da leitegada (GAGGINI, 2017; GARCÍA-VÁZQUEZ et al., 2019), portanto, considera-se esse tipo de anormalidade como critério de reprovação de sêmen de varrão, quando excede $10 \%$ (GAGGINI et al., 2018).

Pode-se observar que o número de animais reprovados reduziu drasticamente no segundo exame, e após as duas análises,
$78 \%$ foram reintroduzidos como reprodutores da central de inseminação. Isso denota a importância da repetição do exame, para reduzir a interferência de alterações espermáticas transitórias no julgamento da permanência de reprodutores doadores de sêmen na central. As alterações morfológicas transitórias podem estar relacionadas a causas traumáticas, alimentares, patológicas, genéticas, iatrogênicas e, ainda, a erros de manipulação da amostra (Brackett, 2006; Arruda et al., 2015), que quando controladas, implicam retorno às taxas de normalidade espermática.

Como no estudo em questão, foram identificados apenas defeitos secundários, ou seja, relacionados a problemas na maturação espermática e ejaculação (gotas proximal e distal), o recomendado seria repetir o exame, considerando o período necessário ao trânsito do espermatozoide pelo epidídimo, que se dá em torno de 15 dias (Anderson, 2004), já se fossem encontrados defeitos primários, a recomendação para a realização da repetição do exame seria em torno de seis a nove semanas, considerando a duração desde a espermatogênese até a maturação epididimária (BRACKETT, 2006). Assim, o exame morfológico deve ser realizado de forma periódica, para observar o grau de evolução das morfopatologias espermáticas 
e avaliar mais precisamente se um varrão deve ser descartado ou não.

Mudanças na morfologia dos espermatozoides também podem ocorrer por alterações na temperatura ambiental e pode variar de acordo com a raça, como em estudo realizado com suínos das raças Duroc e Large White, comparando os valores de anormalidades espermáticas em períodos do ano mais quentes $\left(27-31^{\circ} \mathrm{C}\right)$ e mais frios $\left(15-20^{\circ} \mathrm{C}\right)$, os autores observaram aumento das anormalidades nos períodos mais quentes (KAMANOVA et al., 2021).

Outro fator que influencia na frequência dos defeitos nos espermatozoides suínos é a qualidade do sêmen, principalmente relacionada a concentração espermática. Animais com alta concentração espermática contêm um grande número de espermatozoides e têm um menor volume ejaculado (KONDRACKI et al., 2020). O mesmo estudo observou que em suínos da raça Landrace, a relação entre a concentração espermática e os defeitos morfológicos são mais frequentes quando comparados a raça Large White. Assim, o aumento na concentração espermática reduz a incidência alterações da peça intermediária, porém promove a incidência de espermatozoides com gota citoplasmática proximal ou gota citoplasmática distal (KONDRACKI et al., 2020).

\section{Conclusão}

Através do presente estudo foi possível observar que a avaliação morfológica de espermatozoides em granjas suínas é uma ferramenta extremamente necessária para identificar animais para seleção e descarte reprodutivo, a repetição do exame morfológico em machos que tiveram o sêmen reprovado, devem ser realizadas para não haver falsas conclusões, com o descarte inadequado de reprodutores, já que os defeitos morfológicos podem ser temporários e o animal possa novamente tornar-se apto à reprodução. Futuramente outros estudos devem ser realizados a fim de elucidar acerca das possíveis causas que afetam a morfologia espermática de machos suínos.

\section{Referências Bibliográficas}

ALKMIN, D.V. Central de IA em suínos: Uma análise prática do processo de produção de sêmen de alta qualidade. Rev. Bras. Reprod. Anim., v.43, n.2, p.327-330, abr./jun. 2019

ANDERSON, L.L. Suínos. In: HAZEZ, E.S.E; HAFEZ, B. Reprod. Anim. 7 ed. Manole: São Paulo, p.183-186, 2004.

ARRUDA, R.P.; CELEGHINI, E.C.C.; GARCIA, A.R.; SANTOS, G.C.; LEITE, T. G.; OLIVEIRA, L. Z.; LANÇONI, R.; RODRIGUES, M.P. Morfologia espermática de touros: interpretação $\mathrm{e}$ impacto na fertilidade. Rev. Bras. Reprod. Anim., Belo Horizonte, v.39, n.1, p.47-60, 2015. 
BONET, S.; BRIZ, M.D.; YESTE, M. A Proper Assesment of Boar Sperm Function May Not Only Require Conventional Analyses but Also Others Focused on Molecular Markers of Epididymal Maturation. Reprod Dom Anim, v.47, n. 3, p.52-64, 2012.

BORTOLOZZO, F.P.; WENTZ, I.; BENNEMANN, P.E.; BERNADI, M.L.; WOLLMANN, E.B.; FERREIRA, F.M.; BORCHARDT NETO, G. Suinocultura em ação: Inseminação artificial na suinocultura tecnificada. 1 ed. Porto Alegre: Palloti, 2005. $185 \mathrm{p}$.

BRACKETT, B.G. Reprodução em Mamíferos do Sexo Masculino. In: REECE, W.O. Dukes Fisiologia dos Animais Domésticos. Rio de Janeiro: Guanabara Koogan. 12. ed. cap.38, p.623-643, 2006.

BRIZ, M.D.; BONET, S.; PINART, B.; EGOZCUE, J.; CAMPS, R. Comparative Study of Boar Sperm Coming From the Caput, Corpus, and Cauda Regions of the Epididymis. Journal of Andrology, v.16, n.2, 1995.

BUSTAMANTE-FILHO, I.C.; SOUZA, A.P.B.; LAZARI, L.F.; ARGENTI, L.E.; WEBER, A. Avaliação seminal em suínos: aplicabilidades das analises proteômicas. Rev. Bras. Reprod. Anim., v.43, n.2, p.184-195, abr./jun. 2019.

CAPELETTO, A.; BRAGANÇA, J. F. M.; ROCHA, R. X.; PETROLLI, T. G.; BENNEMANN, P. E. Correlação entre morfologia espermática, taxa de parto e tamanho da leitegada em matrizes suínas. Enciclopédia Biosfera, Centro Científico Conhecer - Goiânia, v.10, n.19; p.627-635, 2014.

Colégio Brasileiro de Reprodução Animal CBRA. Manual para exame andrológico e avaliação de sêmen animal, 3. ed. Belo Horizonte: CBRA, 87 f., 2013.

GAGGINI, T.S. Presença da gota citoplasmática e seus efeitos na morfometria, cromatina e motilidade espermática e identificação de genes candidatos à seleção de parâmetros seminais de suínos. 2017. 134 f. Tese (Doutorado em
Ciências Veterinárias) - Universidade Federal de Uberlândia, Uberlândia, 2017.

GAGGINI, T.S.; BARBOSA, H.; BELETTI, M.E.; REZENDE, F.M.; ANTUNES, R. C. Alta ocorrência de gota citoplasmática distal altera parâmetros relacionados ao movimento espermático de reprodutores suínos. Archives of Veterinary Science, v.23, n.4, p.44-50, 2018.

GARCÍA-VÁZQUEZ，F.A.; HERNÁNDEZCARAVACA, I.; MATÁS, C.; SORIANOÚBEDA, C.; ABRIL-SÁNCHEZ, S.; IZQUIERDO-RICO, M. J. Morphological study of boar sperm during their passage through the female genital tract. Journal of Reproduction and Development, v.61, n.5, 2015.

GARCÍA-VÁZQUEZ, F.A.; MELLAGI, A.P.; ULGUIM, G.; HERNÁNDEZ-CARAVACA, I.; LLAMAS-LÓPEZ, P. J.; BORTOLOZZO, F.P. Post-cervical artificial insemination in porcine: The technique that came to stay. Theriogenology, v.129, n.15, p.37-45, 2019.

GONZALEZ-CADAVID, V. et al. Seminal plasma proteins of adult boars and correlations with

sperm parameters. Theriogenology, v. 82, n. 5, p. 697-707, 2014.

JUNG, M.; RUDIGER, K.; SCHULZE, M. In vitro measures for assessing boar semen fertility. Reprod Domest Anim, v.50, p.20-24, 2015.

KAMANOVA, V.; NEVRKLA, P.; HADAS, Z.; LUJKA, J; FILIPCIK, R. Changes of sperm morphology in Duroc, Landrace and Large White boars depending on the ambient temperature during the year. Czech Academy of Agricultural Sciences, v. 66, p.1-8, 2021.

KNECHT, D.; JANKOWSKA-MAKOSA, A.; DUZINSKI, K. Analysis of the lifetime and culling reasons for AI boars. J Anim Sci Biotech, v.8, p.49, 2017. 
KONDRACKI, S; GÓRSKI, K; IWANINA, M. Impact of sperm concentration on sperm morphology of large white and landrace boars. Livestock Science, v. 241, 2020.

MELLAGI, A.P.G.; QUIRINO, M.; OLIVEIRA, G.S.; GAGGINI, T.S.; PASCHOAL, A.F.L.; LUCCA, M.S.; ULGUIM, R.R.; BORTOLOZZO, F. P. Atualizações na avaliação andrológica em suínos. Rev. Bras. Reprod. Anim., v.43, n.2, p.47-53, abr./jun. 2019.

OBERLANDER, G.; PONTELO, T.P.; MENEZES, T.A.; MURGAS, L.D.S.; ZANGERONIMO, M.G.; SILVA, A.C.; PEREIRA, L.J. Alterações morfológicas encontradas no sêmen de reprodutores suínos (Sus scrofa). Revista Científica Eletrônica de Medicina Veterinária, Ano XI, n.20, 2013.

RODRIGUEZ, A.L. SOOM, A.V.; ARSENAKIS, I. MAES, D. Fatores de manejo e manejo do sêmen afetam a qualidade do sêmen estendido. Porc Health Manag, 2017.
SERTÃO, A.T.; MACHADO, N.F.R. Controle externo da qualidade em espermograma: avaliação do desempenho de laboratórios clínicos participantes de dois provedores de ensaio de proficiência. Brazilian Journal of Clinical Analyses, 2019.

TONIOLLI, R. Aspectos de um programa de inseminação artificial em suínos. Ciência Animal, v.12, n.1, p.7-17, 2002.

TURNER, D.P.; HOULE, T.T. Conducting and reporting descriptive statistics. Headache: The Journal of Head and Face Pain, v. 59, n. 3, p.300-305, 2019.

WABERSKI, D.; MEDING, S.; DIRKSEN, G.; WEITZE, K.F.; LEIDING, C.; HAHN, R. Fertility of long-term-stored boar semen: Influence of extender (Androhep and Kiev), storage time and plasma droplets in the semen. Animal Reproduction Science, v. 36, n.1-2, p.145-151, Jul.1994. 